\title{
Evaluation of a New Digital Automated Glycemic Pattern Detection Tool
}

\author{
María José Comellas, MD, Emma Albiñana, $\mathrm{MD}_{1}^{2}$ Maite Artes, $\mathrm{MSc}_{1}^{3}$ Rosa Corcoy, MD, $\mathrm{PhD}_{1}^{4,5}$ \\ Diego Fernández-García, MD, Jorge García-Alemán, MD, Beatriz García-Cuartero, MD, PhD, \\ Cintia González, MD, PhD, ${ }^{4,5}$ María Teresa Rivero, MD, Núria Casamira, MSc, \\ and Jörg Weissmann, MD, MHBA ${ }^{9}$
}

\begin{abstract}
Background: Blood glucose meters are reliable devices for data collection, providing electronic logs of historical data easier to interpret than handwritten logbooks. Automated tools to analyze these data are necessary to facilitate glucose pattern detection and support treatment adjustment. These tools emerge in a broad variety in a more or less nonevaluated manner. The aim of this study was to compare eDetecta, a new automated pattern detection tool, to nonautomated pattern analysis in terms of time investment, data interpretation, and clinical utility, with the overarching goal to identify early in development and implementation of tool areas of improvement and potential safety risks.

Methods: Multicenter web-based evaluation in which 37 endocrinologists were asked to assess glycemic patterns of 4 real reports ( 2 continuous subcutaneous insulin infusion [CSII] and 2 multiple daily injection $[\mathrm{MDI}])$. Endocrinologist and eDetecta analyses were compared on time spent to analyze each report and agreement on the presence or absence of defined patterns.

Results: eDetecta module markedly reduced the time taken to analyze each case on the basis of the emminens eConecta reports (CSII: $18 \mathrm{~min}$; MDI: 12.5), compared to the automatic eDetecta analysis. Agreement between endocrinologists and eDetecta varied depending on the patterns, with high level of agreement in patterns of glycemic variability. Further analysis of low level of agreement led to identifying areas where algorithms used could be improved to optimize trend pattern identification.

Conclusion: eDetecta was a useful tool for glycemic pattern detection, helping clinicians to reduce time required to review emminens eConecta glycemic reports. No safety risks were identified during the study.
\end{abstract}

Keywords: Blood glucose, Pattern analysis, Diabetes, Pattern management, Automated tool.

\footnotetext{
${ }^{1}$ Roche Diabetes Care Spain SL, Barcelona, Spain.

${ }^{2}$ Vithas Hospital Internacional Medimar, Paediatrics Unit, Alicante, Spain.

${ }^{3}$ Adelphi Spain, Barcelona, Spain.

${ }^{4}$ Hospital de la Santa Creu i Sant Pau, Endocrinology and Nutrition Department, Medicine Department, Universitat Autònoma de Barcelona, Barcelona, Spain.

${ }^{5}$ CIBER-BBN, Zaragoza, Spain.

${ }^{6}$ Hospital Universitario Virgen de la Victoria, Endocrinology and Nutrition Unit, Málaga, Spain.

${ }^{7}$ Hospital Universitario Ramón y Cajal, Pediatric Endocrinology and Diabetes Unit, Madrid, Spain.

${ }_{9}^{8}$ Complejo Hospitalario Universitario de Ourense, Endocrinology and Nutrition Unit, Ourense, Spain.

${ }^{9}$ Roche Diabetes Care Deutschland GmbH, Mannheim, Germany.

This work has been previously presented in the congresses listed below:

- 8th International Conference on Advanced Technologies \& Treatments for Diabetes 2015 (ATTD 2015), Paris, France, February 18-21, 2015.

- ISPOR (International Society for Pharmacoeconomics and Outcomes) 19th Annual European Congress, Vienna, Austria, October 29, 2016 to November 2, 2016.

- XXVII Congreso Nacional de la SED (Sociedad Española de Diabetes), Bilbao, España, April 20-22, 2016.

(C) Maria José Comellas, et al., 2017; Published by Mary Ann Liebert, Inc. This Open Access article is distributed under the terms of the Creative Commons License (http://creativecommons.org/licenses/by/4.0), which permits unrestricted use, distribution, and reproduction in any medium, provided the original work is properly credited.
} 


\section{Introduction}

$\mathbf{H}$ BA1C IS THE GOLD standard as a summary of glycemic control, but regular self-monitoring of blood glucose (SMBG) is the most accessible way to monitor glycemic excursions. However, to adjust the treatment in an appropriate way, an accurate interpretation of the patients' reports is needed, but it is hindered by the large amount of data generated.

Decision-making based on glycemic data is time-consuming and many parameters need to be considered. Pattern management has been defined as the systematic interpretation of SMBG data over time to determine whether changes are required to optimize blood glucose control. ${ }^{1}$ However, patterns are not always easy to detect or interpret and on-meter and web-based tools have been developed to support both patients and clinicians to better interpret the data collected. ${ }^{2}$ Emminens eConecta $^{\circledR}$ is a web-based tool that aims at personalized management of diabetes, where data collected in the devices can be downloaded and reviewed by healthcare professionals (HCPs). Clinicians typically review daily data and trend graphs from the last 4 registered weeks in combination with the electronic logbook and lists to fully understand and match glycemic, carbohydrate, and insulin data coming from pumps and bolus calculators. eDetecta is an emminens eConecta module developed to perform an automatic detection of glycemic patterns based on glycemic, insulin, and carbohydrate data; Figure 1 shows the eDetecta dashboard. The eDetecta module can analyze up to 22 patterns, grouped into 5 blocks: glycemic variability, hypoglycemia, hyperglycemia, use of the system, and adherence to treatment.
Definitions of every pattern are clearly specified in the platform and default values can be easily customized by HCP to allow a personalized pattern analysis. Default configuration was agreed by consensus among a group of endocrinologists and pediatric experts in diabetes. ${ }^{3}$

eDetecta can analyze data provided by glucose meters, bolus calculators, and insulin pumps. An easy-to-interpret dashboard shows if patterns are present (red) or absent (green).

The aim of this study was to compare time requirements and agreement in pattern detection between the automated eDetecta module and nonautomated analysis of the data reports by $\mathrm{HCP}^{4}$

\section{Methods}

\section{Study design}

This multicenter, cross-sectional web-based evaluation included 37 endocrinologists from Spain and Portugal, with an average of 17 years of experience in diabetes. Participants were asked to analyze 4 cases, obtaining a maximum of 145 evaluations; we esteemed it would provide a reasonable statistical power.

The evaluation was organized in three parts: a preliminary questionnaire on eDetecta initial experiences (having used eDetecta in usual clinical practice with at least four patients), an evaluation of reports of real-life data downloads, and a final questionnaire on current barriers related to data analysis in clinical practice.

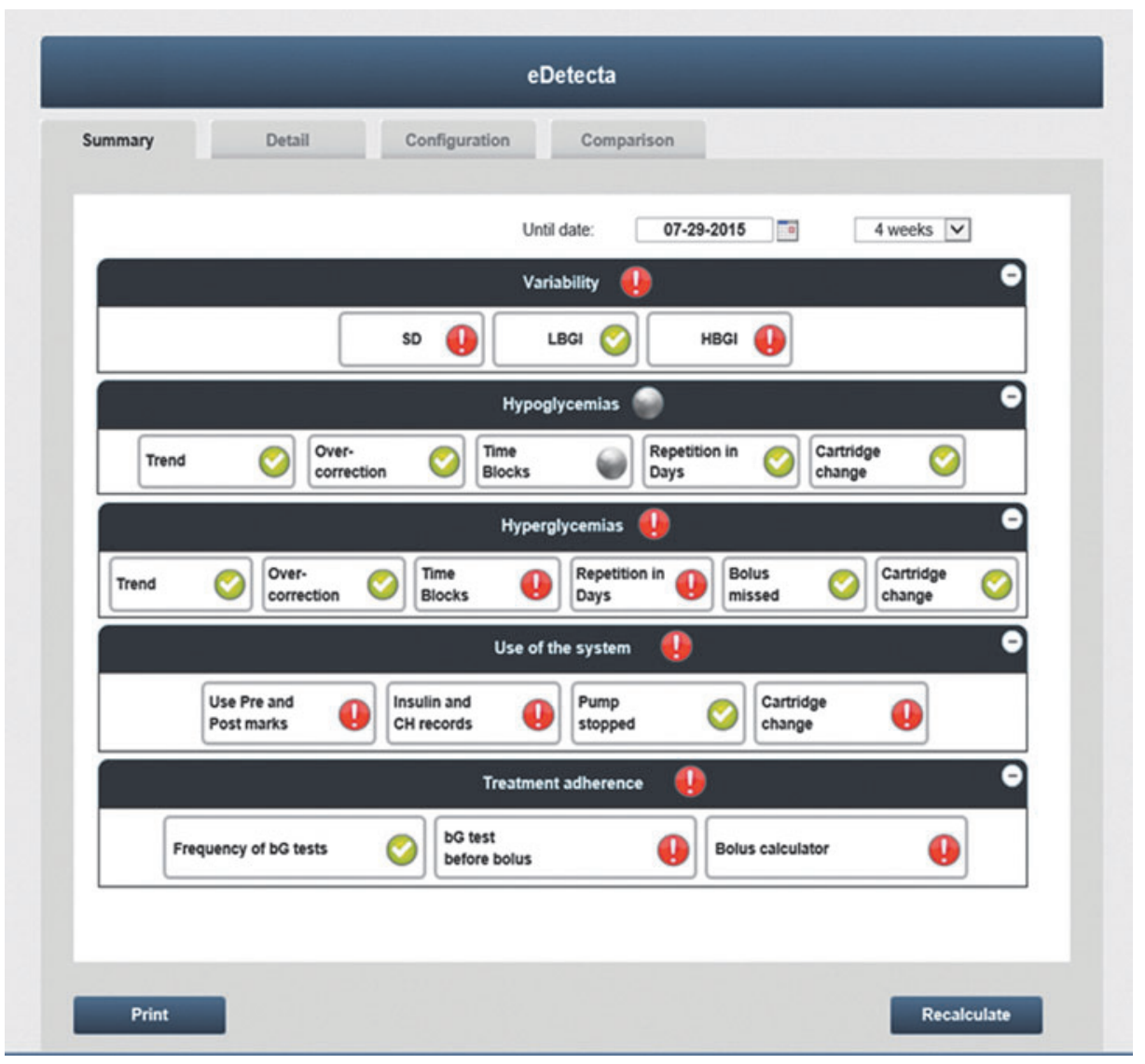

FIG. 1. eDetecta dashboard. 
In the second part of the evaluation, participants were asked to analyze the glycemic patterns of four anonymized reports of real-life patients' data and complete a questionnaire indicating if the patterns available in eDetecta were present or not in the cases. The definition of the eDetecta and all the relevant information needed to perform manual pattern detection were available. The reports had been previously obtained from emminens eConecta platform and included data from two patients using insulin pumps (continuous subcutaneous insulin infusion [CSII] therapy) and two patients using a bolus calculator and multiple daily injections (MDI) to detect different patterns. These reports consisted of tendency graphs, data lists, as well as digital patient logbooks from a period of 4 weeks, representing the reports commonly obtained from the emminens eConecta. Table 1 summarizes the report content for each selected case and an example of the reports obtained, corresponding to the CSII case 2 (Supplementary Data; Supplementary Data are available online at www.liebertpub.com/dia). To minimize any potential bias due to the order in which cases were reviewed, six sequences of the four reports were generated and every participant was randomly assigned to one of them. The access period to the web-based evaluation lasted 4 weeks.

Time spent by participants to analyze each case and answers on presence or absence of predefined patterns were recorded. The clear definition of every pattern was available in the questionnaire.

\section{Data collection}

Total time spent per participant to analyze every report consisted of two separate times: (1) time needed to read the report and (2) time needed to evaluate the presence or absence of patterns and answer the corresponding questions. Time spent by eDetecta on the automated analysis for each one of the four reports was also registered. All times were measured in minutes. Participant responses on the presence of patterns were compared with automated detected patterns by eDetecta. The questionnaire on current barriers related to data analysis in clinical practice included a total number of 30 questions, most of which were based on a 5-point score Likert-type scale (i.e., 1 is "not reliable at all" and 5 is "very reliable").

\section{Statistical analysis}

The level of agreement in pattern detection between HCPs and eDetecta was evaluated using Cohen's kappa coefficient $(\kappa)$ and associated 95\% confidence intervals (CIs) that were cal- culated both overall and for each of the four cases. A $\kappa$ value of 0.81-1.00 indicates almost perfect agreement; a kappa coefficient of $\leq 0.2$ indicates slight or no agreement. The analysis was adjusted by sequence case and country. ${ }^{5}$

The report reading time and patterns evaluation time of the four emminens eConecta reports by $\mathrm{HCP}$ and eDetecta module were evaluated. The total time spent to analyze each case (report reading time+pattern evaluation time) was calculated for the four reports, adjusting by sequence case and country ( $n$, adjusted mean, and $95 \% \mathrm{CI}$ ).

Analysis of opinion questionnaires was conducted by means of absolute and relative frequencies $(n, \%)$ for categorical variables and Likert-scale type variables, and central trend measures ( $n$, mean, standard deviation [SD], median, Q1 and Q3, minimum and maximum, 95\% CI, and mode) for continuous variables. The data analysis was adjusted by sequence case and country.

The data analysis for this article was generated using SAS/ STAT software, Version 9.2 of the SAS System for Windows (Copyright (@) 2002-2008 by SAS Institute, Inc., Cary, NC).

\section{Results}

\section{Participants}

Thirty-seven participants answered the questionnaires and responded on the presence of the patterns; most of the participants were women $(81.1 \%)$, endocrinologists $(83.8 \%)$, and pediatricians $(16.2 \%)$, with a mean time of 17 years of experience in diabetes and visiting a mean number of 18 CSII patients and 68 MDI patients.

\section{Physician opinion about the eDetecta module}

Regarding their experience using the eDetecta module, $91.9 \%$ of the clinicians agreed that eDetecta module recognizes the patterns in a few seconds, $78.3 \%$ concurred that it allows them to make decisions faster, and $72.9 \%$ agreed that it saves time in the clinical evaluation of the patient. $67.5 \%$ of the HCP agreed that the module facilitates a more accurate identification of the abnormal patterns present in the data downloaded, and $62.1 \%$ agreed that it provides reassurance regarding the detection of anomalies. $75.7 \%$ agreed that the module allows making more adequate decisions and $70.2 \%$ agreed that it allows to dedicate more time to the patient. Finally, 59.4\% of the participants agreed that the module recognizes a comprehensive list of patterns (Fig. 2).

Regarding the usefulness of the eDetecta module, physicians think that it is useful in automatic pattern detection.

Table 1. Emminens eConecta Reports Content and Time Recovered for Each of the Four Cases Used to Evaluate the eDetecta Module

\begin{tabular}{|c|c|c|c|c|c|c|c|c|}
\hline & \multicolumn{4}{|c|}{ Graphs } & \multicolumn{3}{|c|}{ Lists } & \multirow[b]{2}{*}{$\begin{array}{c}\text { Time } \\
\text { (weeks) }\end{array}$} \\
\hline & Days & Weeks & Daily trend & Trend & Statistics & $\begin{array}{c}\text { Combined }^{\mathrm{a}} \\
\text { data (pages) }\end{array}$ & $\begin{array}{c}\text { Combined } \\
\text { logbook (pages) }\end{array}$ & \\
\hline Case 1 CSII & Half page & Half page & One page & Half page & One page & 42 & 7 & 4 \\
\hline Case 2 CSII & Half page & Half page & One page & Half page & One page & 27 & 4 & 2 \\
\hline Case 1 MDI & Half page & Half page & & Half page & One page & 10 & 6 & 4 \\
\hline Case 2 MDI & Half page & Half page & & Half page & One page & 8 & 5 & 4 \\
\hline
\end{tabular}

${ }^{\mathrm{a}}$ Combined: glycemic, $\mathrm{CH}$, insulin and device data at the same list.

$\mathrm{CH}$, carbohydrate; CSII, continuous subcutaneous insulin infusion; MDI, multiple daily injections. 


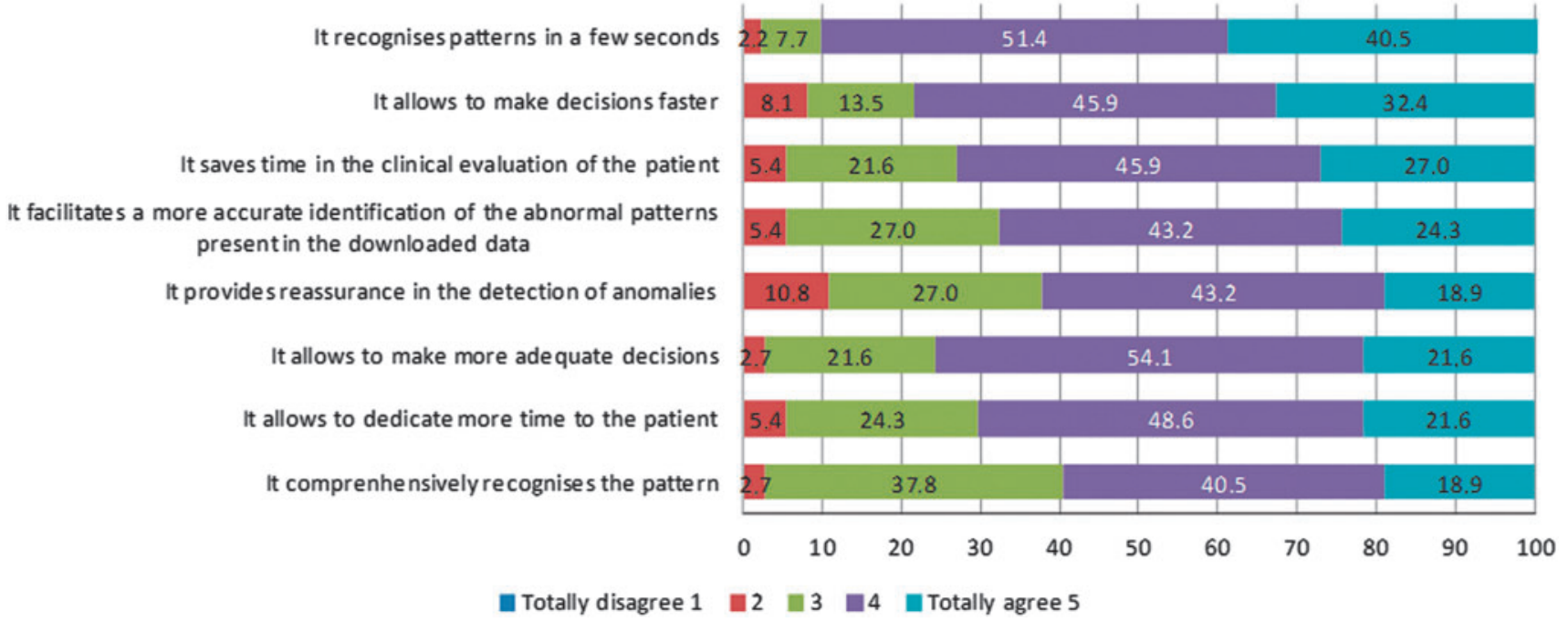

FIG. 2. Physician opinion regarding the experience using the eDetecta module.

94.6\% agreed that its color code facilitates the interpretation of the data; $83.7 \%$ agreed that it is useful to compare between periods of time; $89.1 \%$ appreciate having a customizable configuration for each individual patient; $83.7 \%$ appreciate the visualization of all the patterns at the same time, and $64.8 \%$ appreciate the analysis detail, including the data that generated the alerts (Fig. 3).

$97.3 \%$ of the participants agreed that, when changes in the configuration are needed, the available ranges cover the more frequent clinical situations. $83.7 \%$ of the participants agreed that the default configuration of the different patterns is applicable to most of the patients.

In their usual practice, $70.3 \%$ of the participating physicians kept the default configuration in all the patterns of the eDetecta module, while $29.7 \%$ changed some of them, mainly hypoglycemia (90.9\%) and hyperglycemia (72.7\%). Pregnancy was the main reason to change pattern configuration in all the patterns changed.

Participants were also asked about missed relevant patterns in the module and the answer was "no" for $89.2 \%$ of them.

\section{Analysis of the cases}

To evaluate the eDetecta module, each investigator was asked to analyze the report corresponding to 4 cases; a maximum of 145 evaluations was obtained. CSII cases 1 and 2 were reviewed by 35 and 36 participants, respectively. All 37 endocrinologists reviewed the 2 cases under MDI therapy.

Time spent to analyze the cases. Mean time spent by physicians to analyze CSII cases was $17 \mathrm{~min}$ for case $1(95 \%$ CI: 13.3-19.3) and $19 \mathrm{~min}$ for case 2 (95\% CI: 15.6-22.2). For the MDI cases, mean time to complete case analysis was 12 min for case 1 (95\% CI: 9.0-15.5) and 13 min for case 2 (95\% CI: 9.3-15.8).

In contrast, the eDetecta module performed the pattern analysis automatically.

Global pattern detection. The comparison between the detection of the 19 patterns by HCP and the new eDetecta module showed a variable agreement depending on the case

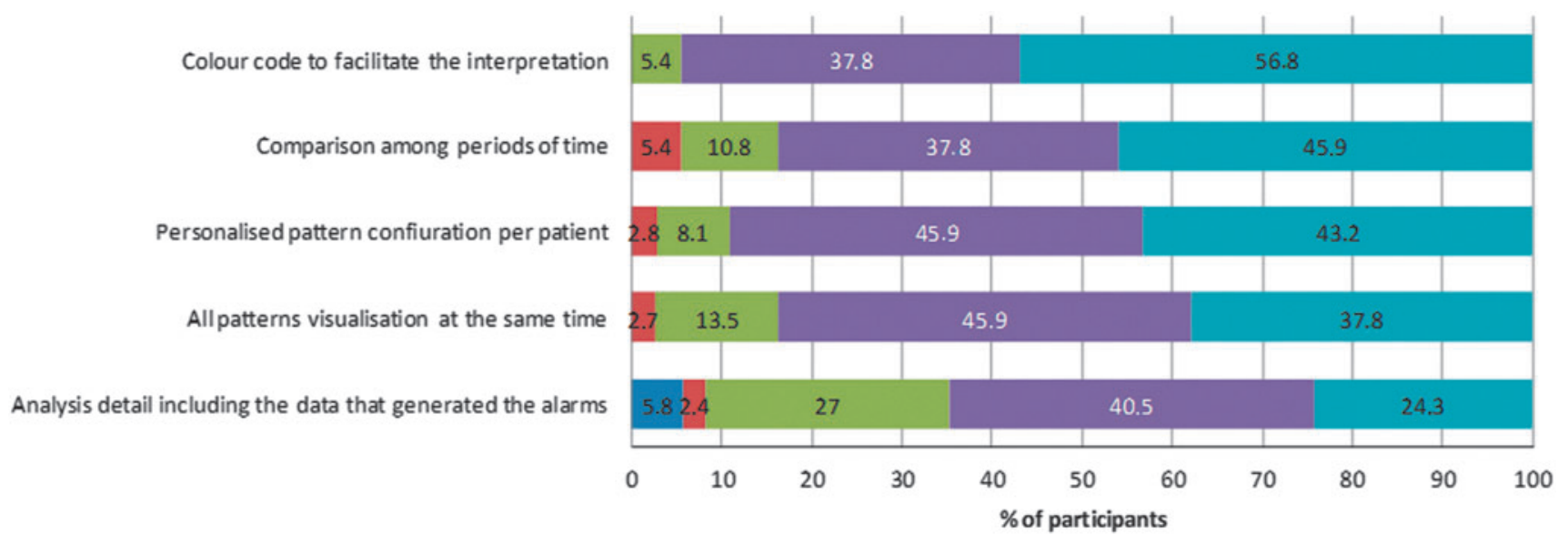

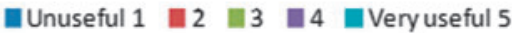

FIG. 3. Physician perception of the usefulness of the eDetecta module. 
and existing patterns. Overall, the agreement was high $(>65 \%$ of agreement in 16 patterns).

Table 2 provides the glycemic patterns defined by default in the eDetecta module.

Table 3 illustrates the patterns identified by the eDetecta module and level of agreement with the physician analysis (present/absent), both overall and for each individual pattern across the four cases.

Variability patterns. The overall level of agreement for the variability patterns was high. Presence/absence of the individual patterns was identified in agreement with eDetecta by $95.9 \%$ of physicians for SD, $89.7 \%$ for low blood glucose index, and $91.7 \%$ for high blood glucose index with $\kappa$ values ranging from 0.62 to 0.88 (Table 2).

Hypoglycemia patterns. Across all four cases, identification of the presence/absence of "hypoglycemic trend," "overcorrection hypoglycemia," "hypoglycemia in a defined time block," and "hypoglycemia associated with a cartridge change" was globally in agreement with eDetecta by $66.9 \%, 63.4 \%, 78.6 \%$, and $97.2 \%$, respectively.

Regarding "hypoglycemic trend" (see Table 1 for pattern definition), the highest level of agreement was achieved for the MDI case 1 , where $89.2 \%$ of the participants identified the present pattern, and the lowest level of agreement was achieved in the MDI case 2, where the pattern was not present, but identified by $27.0 \%$ of the clinicians.
For "hypoglycemia due to overcorrection," the highest level of agreement was obtained in the CSII case 1 (91.4\%), where the pattern was not present, and the lowest level of agreement was obtained in the CSII case 2, where the pattern was present and identified by $33.3 \%$ of the clinicians.

In the pattern "hypoglycemia within a defined time block," agreement ranged from $97.3 \%$ for the MDI case 1 to $55.6 \%$ for the CSII case 2, being the pattern present in both cases.

"Hypoglycemia associated with a cartridge change" was the pattern with the highest global agreement, reaching 94.3\% in the CSII case 1 and $100 \%$ in the CSII case 2, being the pattern absent in both cases.

Hyperglycemia patterns. With regard to hyperglycemia patterns, global agreement was $44.1 \%$ for "hyperglycemic trend," $69.0 \%$ for "cartridge change delay" and "overcorrection hyperglycemia," $74.5 \%$ for "hyperglycemia in time block," and $80.3 \%$ for "missed bolus."

For "hyperglycemic trend," the highest level of agreement was observed in the CSII case 2, where $91.7 \%$ of the participants recognized the present pattern, and the lowest level of agreement was observed in the CSII case 1 , where the pattern was absent, but recognized only by $2.9 \%$ of the clinicians.

Regarding "hyperglycemia due to cartridge change delay," the level of agreement was $60.0 \%$ for the CSII case 1 and $77.8 \%$ for the CSII case 2. In both cases, the pattern was not present.

Table 2. Glycemic Patterns Included in the Automated eDetecta Module

\begin{tabular}{|c|c|c|}
\hline Blocks & Patterns & Pattern definition \\
\hline Variability & $\begin{array}{l}\text { Standard deviation } \\
\text { Low Blood Glucose Index } \\
\text { High Blood Glucose Index }\end{array}$ & $\begin{array}{l}>70 \mathrm{mg} / \mathrm{dL} \\
\text { Moderate or high risk of severe hypoglycemia } \\
\text { Moderate or high risk of severe hyperglycemia }\end{array}$ \\
\hline $\begin{array}{l}\text { Hypoglycemia } \\
\text { (glycemia } \\
<70 \mathrm{mg} / \mathrm{dL} \text { ) }\end{array}$ & $\begin{array}{l}\text { Hypoglycemic trend } \\
\text { Overcorrection hypoglycemia } \\
\text { Hypoglycemia in a defined } \\
\text { time block } \\
\text { Associated with a cartridge } \\
\text { change }^{\mathrm{a}}\end{array}$ & $\begin{array}{l}>1 \text { Hypoglycemic event/day on } 3 \text { consecutive days } \\
>25 \% \text { Hypoglycemic events preceded by a hyperglycemic event } \\
\text { Before/after breakfast, lunch, evening meal, bedtime, nocturnal } \\
\text { Hypoglycemia present in }>80 \% \text { of cartridge changes }\end{array}$ \\
\hline $\begin{array}{l}\text { Hyperglycemia } \\
\quad(>160 \mathrm{mg} / \mathrm{dL})\end{array}$ & $\begin{array}{l}\text { Hyperglycemic trend } \\
\text { Associated with cartridge } \\
\text { change delay }^{\mathrm{a}} \\
\text { Overcorrection hyperglycemia } \\
\text { Hyperglycemia in time block } \\
\text { Missed bolus }^{\mathrm{a}}\end{array}$ & $\begin{array}{l}>2 \text { Hyperglycemias/day for } 7 \text { consecutive days } \\
\text { Cartridge change delay of }>1 \text { day during which the }>50 \% \\
\text { glycemic values are in the hyperglycemia range } \\
>25 \% \text { of detected hyperglycemias (glycemia }>200 \mathrm{mg} / \mathrm{dL} \text { ) } \\
\text { are preceded by a hypoglycemia within }<3 \mathrm{~h} \\
\text { Over } 7 \text { days, }>3 \text { hyperglycemias in the same time block } \\
\text { (preprandial glycemia }>150 \mathrm{mg} / \mathrm{dL} \text {, postprandial } \\
\text { glycemia }>180 \mathrm{mg} / \mathrm{dL} \text { ) } \\
\text { Over } 4 \text { weeks, }>3 \text { hyperglycemias related to missed bolus } \\
\text { (intake of }>20 \mathrm{~g} \text { of } \mathrm{CH} \text { unrelated to a hypoglycemia } \\
\text { and without record of insulin bolus in the } 2 \mathrm{~h} \text { before } \\
\text { the hyperglycemia) }\end{array}$ \\
\hline $\begin{array}{l}\text { Use of the } \\
\text { system }\end{array}$ & $\begin{array}{l}\text { Use of premarks and postmarks } \\
\text { Insulin and } \mathrm{CH} \text { record } \\
\text { Pump stopped } \\
\text { Cartridge change (every } 7 \text { days) })^{\mathrm{a}}\end{array}$ & $\begin{array}{l}<3 \text { Glycemias/day with preprandial or postprandial } \\
\text { marking on }>50 \% \text { of days } \\
<3 \text { Insulin and carbohydrate records per day } \\
>2 \text { Stops a day on }>50 \% \text { of days } \\
\text { Cartridge change delay in }>30 \% \text { of changes }\end{array}$ \\
\hline $\begin{array}{l}\text { Treatment } \\
\text { adherence }\end{array}$ & $\begin{array}{l}\text { Frequency of BG tests } \\
\text { BG test before bolus } \\
\text { Bolus calculator use }^{\mathrm{a}}\end{array}$ & $\begin{array}{l}<4 \text { Measurements/day on } 80 \% \text { of days } \\
\text { In }>25 \% \text { of boluses, no glycemia measurement in previous } 30 \mathrm{~min} \\
\text { The bolus calculator was not used in }>25 \% \text { of the boluses }\end{array}$ \\
\hline
\end{tabular}

${ }^{\mathrm{a} C}$ Continuous insulin infusion cases only.

BG, blood glucose. 


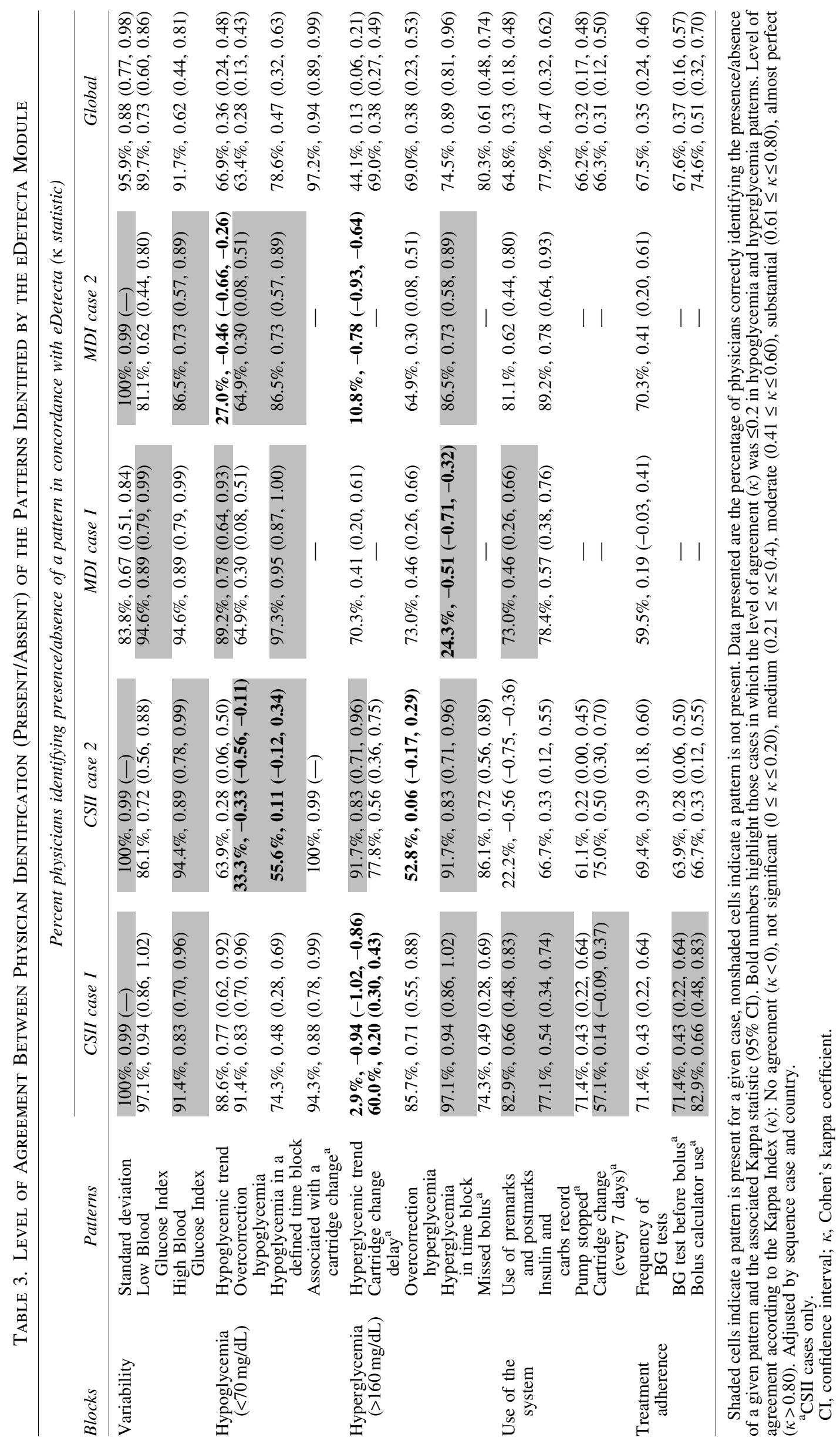


For "hyperglycemia due to overcorrection," the highest and lowest levels of agreement were observed in the CSII cases, ranging from $85.7 \%$ in the case 1 to $52.8 \%$ in the case 2 , being the pattern absent in both cases.

For "hyperglycemia in time block," the highest level of agreement was found in the CSII case 1, where the pattern was present and correctly identified by $97.1 \%$ of the clinicians, and the lowest level of agreement was observed in the MDI case 1, being the pattern present and identified by only $24.3 \%$ of the participants.

In the case of "hyperglycemia due to missed bolus," the level of agreement was $74.3 \%$ for the CSII case 1 and $86.1 \%$ for the CSII case 2, being the pattern absent in both cases.

Use of system patterns. Global agreement for "use of system" patterns was moderate for the four patterns included. Regarding the individual patterns, the level of agreement was moderate-good in three of the four cases for the "preprandial and postprandial marks" pattern and in all four cases for the "insulin and carbohydrate [CH]" pattern, it was moderate in both CSII cases for "pump stopped," and for "cartridge change," it was poor in CSII case 1 and moderate in CSII case 2 .

Treatment adherence patterns. Global agreement was moderate for all three treatment adherence patterns. Pattern "frequency of BG tests," which was absent in all four cases, was identified in agreement with eDetecta by $71.4 \%$ of the physicians for CSII case $1,69.4 \%$ of the physicians for CSII case $2,59.5 \%$ of the physicians for MDI case 1 , and $70.3 \%$ of the physicians for MDI case 2. Level of agreement was moderate-good for the patterns "BG test before bolus" and "bolus calculator use" with a global agreement of $67.3 \%$ and $74.6 \%$, respectively, assessed for the two CSII cases.

\section{Physician opinion about data analysis}

Regarding the frequency of use of data review methods, participating physicians suggested that data management tools are mainly used to review data in patients with insulin pumps $(84.1 \%)$ and bolus calculators (79.2\%). In contrast, these tools are only used in $39.6 \%$ of patients using a blood glucose meter, the manual review of patient logbooks being the most commonly used method.

When asked about the barriers found in using these tools, physicians highlighted time spent installing different software for different devices as the main difficulty.

They indicated that in clinical practice more time is needed to review insulin pump data (estimated mean time $20.5 \mathrm{~min}$ ), followed by bolus calculator data (mean $14.7 \mathrm{~min}$ ) and meter data (mean $11.5 \mathrm{~min}$ ). In summary, physicians found the eDetecta module useful for automatic pattern detection. The majority of physicians (94.6\%) indicated that it was a reliable tool. $83.8 \%$ of physicians were generally satisfied with the module and $89.2 \%$ of physicians would recommend emminens eConecta with the eDetecta module to their colleagues.

\section{Discussion}

In this case- and survey-based evaluation of glycemic pattern recognition with the eDetecta module, good level of agreement was shown between physicians and eDetecta pattern recognition, and physicians reported this tool to be useful, reliable, and comprehensive for clinical practice. Previous studies have demonstrated the utility of pattern detection software in achieving more rapid and accurate pattern detection compared with nonautomated review of clinical data. ${ }^{6,7}$

Good level of agreement was shown between physician and eDetecta pattern recognition for patterns relating to glycemic variability. In these cases, the eDetecta module could prevent time spent by physicians on mental calculations to identify clinically relevant patterns. The module can detect automatically moderate or high ranges of Low Blood Glucose Index and High Blood Glucose Index, and thresholds for SD and percentage coefficient of variation for glucose $(\% \mathrm{CV}$, obtained from the calculation: [SD of glucose/mean glucose] $\times 100$ ) can be configured. Having a glycemic variability pattern related to $\% \mathrm{CV}$ can facilitate an easy identification of stable or unstable glycemic status. In a recent study, a relationship between the \% $\mathrm{CV}$ and frequency of hypoglycemia was found, which was significantly greater in subjects who had a $\mathrm{CV}$ value $>36 \%$ than in those who were below this threshold. ${ }^{8}$ In the other patterns (hypoglycemia, hyperglycemia, use of the system, and treatment adherence), low level of agreement was observed in at least one of the cases, but low level of agreement in the same pattern was never present for all four cases.

Patterns of particular clinical relevance where the level of agreement between the eDetecta pattern identification and clinical opinion was low included the following: "hypoglycemic/hyperglycemic trends," "overcorrection-associated hypoglycemia/hyperglycemia," and identification of "hypoglycemic/hyperglycemic patterns within defined time blocks." A deeper analysis focusing on the results with low level of agreement was conducted.

One of the main causes of low level of agreement was the understanding of the provided definition for every pattern. For example, "Hypoglycemic Trend" was initially defined as "more than 1 hypoglycemia per day over 3 consecutive days." This definition led to a low level of agreement $(\kappa=-0.46)$ in MDI case 2 , where only $27 \%$ of participants considered that the trend was present. According to participants, "Hypoglycemia Trend" definition would be more clinically relevant if " $>1$ " was changed to "at least 1 or more hypoglycemias per day over 3 consecutive days," potentially improving agreement. Similarly, when "Hyperglycemic trend" is defined as "more than 2 hyperglycemias a day for 7 consecutive days," a low level of agreement is obtained in MDI case $2(\kappa=-0.78)$; the authors suggested to change " $>2$ " for " 2 or more hyperglycemias a day for 4 consecutive days," possibly increasing agreement, since this would have clinical sense.

Pattern definition was reached by consensus of a scientific committee ${ }^{3}$ composed by a group of endocrinologists and pediatric experts in diabetes, with the aim to cover most patients and signal those situations that need attention according to clinical experience; however, the results showed that some of the default pattern configurations do not fit with the usual clinical practice. That is why it is important to highlight that eDetecta pattern definitions are customizable to adjust the proper values for every patient or group of patients by the HCPs.

Low level of agreement was also present in patterns where definitions involved a rate like "overcorrection hypoglycemia and hyperglycemia" (Table 2). Regarding "hypoglycemia by hyperglycemia overcorrection," the low level of agreement could be explained due to the difficulty in mentally calculating the $25 \%$ of cases preceded by a hyperglycemic event, as 
described in the pattern definition. The same pattern in MDI case 2 reached a higher percentage of identification because the ratio between the hypoglycemias and previous hyperglycemias was more evident.

When analyzing the low level of agreement in the CSII case 2 for "hyperglycemia by hypoglycemia overcorrection," the proposed definition did not completely match the perception of the clinicians. According to the authors, the definition would be more clinically relevant if the denominator of the equation is not the total number of hyperglycemias and the threshold (glycemia $>200 \mathrm{mg} / \mathrm{dL}$ ) is higher, potentially improving agreement.

Finally, low level of agreement was also present in cases where contrasting patterns were present. For example, in MDI case 1, the level of agreement in the pattern "Hyperglycemia in Time Block" was low $(\kappa=-0.51)$. In this report, there was a predominant hypoglycemic context at the same time. For physicians, "Hyperglycemia in Time Block" was not relevant in the presence of "Hypoglycemia in Time Block" and therefore, the level of agreement with eDetecta was low. However, eDetecta did actually signal the presence of both patterns.

The use of this type of pattern detection software can also provide caregivers further information on the use of devices, which is closely related to therapy adherence and is difficult to obtain otherwise. Patterns related to the frequency of blood glucose tests before bolus and the use of bolus calculators had a good level of agreement and were appreciated by the participants. Future studies will show if the automatic tool eDetecta has a clinical impact in diabetes management.

This evaluation was conducted in a version only for professional use. The future availability of a version for patient use could help the empowerment of the patient, and probably improve the knowledge of the disease and promote self-care behaviors in their daily life. ${ }^{9}$

The results of this research have demonstrated the clinical utility and acceptability of the eDetecta module to support physicians in evaluating real-life glucose downloads, both in terms of time invested and pattern identification. This module is already in use in different centers in Spain and Portugal; more ranges in pattern definition will be configured in the module to include as many patients as possible in the default values, although the capability to adjust the alerts to the needs of every patient or group of patients by the clinicians will remain as one of the features of the module, allowing a personalized management of the patient with diabetes.

\section{Acknowledgments}

The authors acknowledge all the participants who have collaborated in the accomplishment of this study.

\section{Author Disclosure Statement}

M.J.C. and N.C. are employees of Roche Diabetes Care, Spain. E.A. has received speaker fees, consultancy fees, and/ or educational sponsorships from Eli Lilly and Company, Boehringer-Ingelheim, and Roche Diabetes Care. M.A. is the managing director of Adelphi Spain. R.C. has received speaker fees, consultancy fees, and/or educational sponsorships from Ferrer, Eli Lilly and Company, Medtronic, Novo Nordisk, Roche Diabetes Care, and Sanofi-Aventis. D.F.-G. has received speaker fees, consultancy fees, and/or educational sponsorships from Novo Nordisk, Roche Diabetes
Care, Eli Lilly and Company, Boehringer-Ingelheim, Abbott Diabetes Care, Ascensia Diabetes Care, Johnson and Johnson, Astra-Zeneca, Servier, and Amgen. J.G.-A. has received speaker fees, consultancy fees, and/or educational sponsorships from Eli Lilly and Company, Boehringer-Ingelheim, Novo Nordisk, Roche Diabetes Care, Abbott Diabetes Care, Ascensia Diabetes Care, Johnson and Johnson, and AstraZeneca. B.G.-C. has received speaker fees, consultancy fees, and/or educational sponsorships from Novo Nordisk, SanofiAventis, and Roche Diabetes Care. C.G. has received speaker fees, consultancy fees, and/or educational sponsorships from Eli Lilly and Company, Boehringer-Ingelheim, Roche Diabetes Care, Abbott, and Sanofi-Aventis. M.T.R. has received speaker fees, consultancy fees, and/or educational sponsorships from AstraZeneca, Novo Nordisk, Esteve, Ferrer, Sanofi-Aventis, and Roche Diabetes Care. J.W. is an employee of Roche Diabetes Care Deutschland $\mathrm{GmbH}$.

\section{References}

1. Hirsch IB, Bode BW, Childs BP, et al.: Self-monitoring of blood glucose (SMBG) in insulin- and non-insulin-using adults with diabetes: consensus recommendations for improving SMBG accuracy, utilization, and research. Diabetes Technol Ther 2008;10:419-439.

2. Choudhary P, Genovese S, Reach G: Blood glucose pattern management in diabetes: creating order from disorder. J Diabetes Sci Technol 2013;7:1575-1584.

3. Comellas MJ, Albiñana E, Corcoy R, et al.: Consensus to define alert values of a pattern detection web based module. Diabetes Technol Ther 2016;18(Suppl. 1):A1-A140. Presented at 9th International Conference on Advanced Technologies \& Treatments for Diabetes (ATTD), February 2016, Milan, Italy.

4. Comellas MJ, González-Blanco C, García-Alemán J, et al.: Methodology of the evaluation of the new emminens eConecta eDetect module. Poster session presented at 8th International Conference on Advanced Technologies \& Treatments for Diabetes, February 18-21, 2015, Paris, France.

5. Carrasco JL, Jover L: Métodos estadísticos para evaluar la concordancia. [Statistical approaches to evaluate agreement.] Med Clin (Barc) 2004;122(Suppl. 1):28-34.

6. Grady M, Campbell D, MacLeod K, Srinivasan A: Evaluation of a blood glucose monitoring system with automatic high and low-pattern recognition software in insulin-using patients: pattern detection and patient-reported insights. J Diabetes Sci Technol 2013;7:970-978.

7. Katz LB, Dirani RG, Li G, et al.: Automated glycemic pattern analysis can improve health care professional efficiency and accuracy. J Diabetes Sci Technol 2013;7:163-166.

8. Monnier L, Colette C, Wojtusciszyn A, et al.: Towards defining the threshold between low and high glucose variability in diabetes. Diabetes Care 2017;40:832-838.

9. Hernandez-Tejada MA, Campbell JA, Walker RJ, et al.: Diabetes empowerment, medication adherence and self-care behaviors in adults with type 2 diabetes. Diabetes Technol Ther 2012;14:630-634.

Address correspondence to: María José Comellas, MD Roche Diabetes Care Spain SL 08174 Sant Cugat del Vallés Spain

E-mail: maria-jose.comellas@ roche.com 